\title{
Assessing the quality of published genetic association studies in meta-analyses: the quality of genetic studies (Q-Genie) tool
}

\author{
Zahra N. Sohani ${ }^{1,2}$, David Meyre ${ }^{1,2,3}$, Russell J. de Souza ${ }^{1,2}$, Philip G. Joseph ${ }^{4}$, Mandark Gandhi ${ }^{5}$, Brittany B. Dennis ${ }^{1,2}$, \\ Geoff Norman ${ }^{6}$ and Sonia S. Anand ${ }^{1,2,4^{*}}$
}

\begin{abstract}
Background: Advances in genomics technology have led to a dramatic increase in the number of published genetic association studies. Systematic reviews and meta-analyses are a common method of synthesizing findings and providing reliable estimates of the effect of a genetic variant on a trait of interest. However, summary estimates are subject to bias due to the varying methodological quality of individual studies. We embarked on an effort to develop and evaluate a tool that assesses the quality of published genetic association studies. Performance characteristics (i.e. validity, reliability, and item discrimination) were evaluated using a sample of thirty studies randomly selected from a previously conducted systematic review.
\end{abstract}

Results: The tool demonstrates excellent psychometric properties and generates a quality score for each study with corresponding ratings of 'low', 'moderate', or 'high' quality. We applied our tool to a published systematic review to exclude studies of low quality, and found a decrease in heterogeneity and an increase in precision of summary estimates.

Conclusion: This tool can be used in systematic reviews to inform the selection of studies for inclusion, to conduct sensitivity analyses, and to perform meta-regressions.

Keywords: Quality assessment, Genetic association studies, Genetic epidemiology

\section{Background}

Completion of the human genome project along with rapid advances in genotyping technology has resulted in an increase in the number of published genetic association studies (Additional file 1: Figure S1) [1].

Systematic reviews and meta-analyses are a common approach to synthesizing these data. However, in combining studies, authors must consider potential limitations and biases introduced by included studies. In addition to the challenges common to classical epidemiological designs (i.e. sampling error, confounding, and selective reporting), genetic association studies face additional unique threats to validity (Table 1). Notably, because a vast majority of genotype-phenotype associations have modest effect sizes, genetic studies must be

\footnotetext{
*Correspondence: anands@mcmaster.ca

'Population Genomics Program, Department of Clinical Epidemiology and Biostatistics, McMaster University, Hamilton, ON, Canada

${ }^{2}$ Chanchlani Research Centre, McMaster University, Hamilton, ON, Canada Full list of author information is available at the end of the article
}

appropriately powered, often having sample sizes of thousands of subjects. Additional threats to validity include i) quality of genotyping, ii) batch related differences in genotyping, which can manifest as false associations if all cases are in one batch and controls are in the other, iii) choice of inheritance model, and iv) genotype-phenotype relationships confounded by genegene and gene-environment interactions [1-3]. Ultimately, inferences from genetic association studies require careful assessment of traditional epidemiologic biases as well as genetic specific threats to validity.

Several guidelines have been published to guide the conduct and reporting of genetic association studies [3-8]. Among the most notable are the Strengthening the Reporting of Genetic Association Studies (STREGA) and Strengthening the Reporting of Genetic Risk Prediction Studies (GRIPS) statements. Furthermore, the Human Genome Epidemiology Network (HuGENet) Working Group developed a grading scheme to aid researchers in 
Table 1 Common bias in genetic association studies

\begin{tabular}{ll}
\hline Bias & Impact on results of genetic association study \\
\hline Phenotype definition & $\begin{array}{l}\text { Unclear definition of phenotype or use of non-standardized definitions can lead to noise in the outcome, which } \\
\text { compromises ability to identify corresponding susceptibility variants. }\end{array}$ \\
$\begin{array}{l}\text { Genotyping } \\
\text { misclassification }\end{array}$ & $\begin{array}{l}\text { Differential misclassification of genotypes can positively or negatively affect associations depending on the direction of } \\
\text { misclassification. Non-differential misclassification of genotypes will bias association toward the null. }\end{array}$ \\
Selection of sample & $\begin{array}{l}\text { Source of cases and controls or participants for analysis of quantitative traits can bias the association; for example, } \\
\text { contrasting hospital cases with controls from the general population will inflate the association. } \\
\text { Confounding by ethnic } \\
\text { origin }\end{array}$ \\
If populations from ethnic groups differ in frequency of risk alleles, confounding may occur if the populations are unevenly \\
distributed across comparison groups. \\
Relatedness & $\begin{array}{l}\text { Testing a multitude of genetic variants against a phenotype creates a possibility of finding significant associations by } \\
\text { chance (type 1 error). } \\
\text { Consanguinity in genetic association studies can distort the genotype-phenotype associations. Even in supposed unrelated } \\
\text { populations, some individuals may be related. Relatedness should therefore be investigated with additional methods and } \\
\text { adjusted for in the statistical analysis. } \\
\text { The phenotype under investigation may be modified by treatments and hence distort the size of association between } \\
\text { genetic variants and the phenotype of interest. }\end{array}$ \\
\hline
\end{tabular}

assessing the credibility of genetic epidemiological evidence based on three criteria: i) amount of evidence, ii) replication, and iii) protection from bias [2]. Each study is marked as 'A', ' $\mathrm{B}$ ', or ' $\mathrm{C}$ ' based on the strength of evidence on the three criteria and a cumulative rating is then obtained using different combinations. While the scheme provides a good baseline to assess evidence in genetic association studies, it is not intuitive to use, and relies on a checklist approach, which has been shown in literature to be less reliable than global rating scales [9]. Moreover, to our knowledge, the grading scheme itself has not been formally tested for validity and reliability.

In this paper, we: i) describe the development of a tool to assess global quality of published genetic association studies, ii) evaluate the tool's reliability and validity, and iii) investigate whether the reliability and validity of the tool differs based on user's familiarity with genetic association studies, since there is some evidence to suggest that experts outperform novices on evaluations involving knowledge across different content areas [10-13].

\section{Methods}

\section{Development of the Q-Genie tool}

Published guidelines and recommendations on appropriate conduct of genetic association studies, including the STREGA and GRIPS guidelines as well as recommendations by Human Molecular Genetics, Diabetologia, Nature Genetics, and individual research groups [3, 5, 7, 8, 14], were used to create a list of items with potential impact on quality. The items were divided into nine categories: rationale for study, selection of sample, classification of exposure, classification of outcome, sources of bias, presentation of statistical plan, quality of statistical methods, testing of assumptions made in genetic studies, and interpretation of results. The categories were then formulated into questions and a description was included to provide context for each question. A Likert type rating scale was created with seven categories anchored by 'poor' and 'excellent' to ensure minimum loss of precision and reliability and to account for end aversion bias [15]. Additionally, the positive side of the scale was expanded to account for positive skew bias (a tendency to select responses on the favorable end of the scale leading to a ceiling effect in positive ratings) [15]. The final scale used in our tool is depicted in Fig. 1.

A preliminary draft of the tool was sent to five experts with experience in conducting genetic association studies and knowledge in developing measurement tools. The experts were asked to provide suggestions for improvement and comment on the clarity of the items. Discussion with the experts prompted addition of the following aspects lacking from the preliminary draft of the tool: i) checking for samples with outlying heterozygosity, ii) checking both sample and genetic variant missingness, iii) randomization of samples at genotyping stage, iv) checking for concordance of reported sex with genetically determined sex, v) concordance of reported ethnicity with genetically determined ethnicity, and vi) sample size/power considerations. Additionally, the question on classification of the genetic variant was split into two questions, technical and nontechnical classification, respectively.

\section{Psychometric assessment}

We tested the validity and reliability of the Q-Genie tool using a sample of thirty studies randomly selected from a previously conducted systematic review on the association of single nucleotide polymorphisms with type 2 diabetes mellitus in South Asians [16]. Characteristics of the included studies are presented in Additional file 1: Table S1. We used this published systematic review as our sampling frame, instead of a random selection of published studies from scientific databases (e.g. MEDLINE), to ensure 


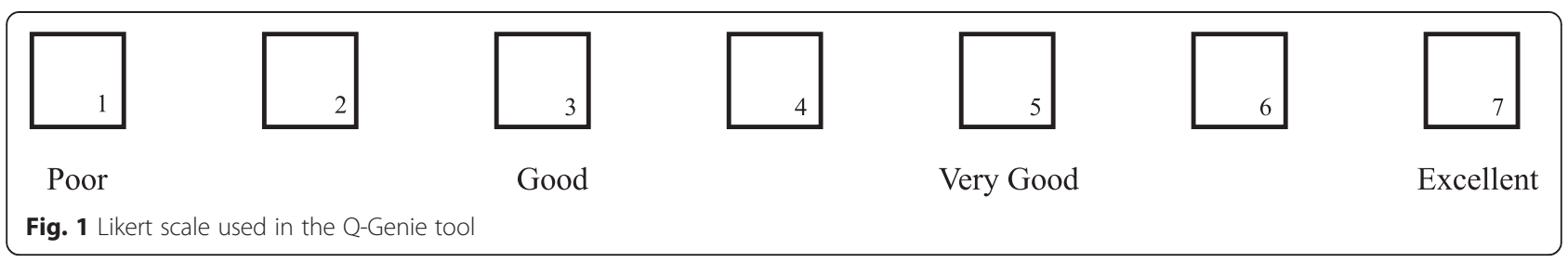

generalizability, since the tool is intended for use in systematic reviews.

Four raters, 2 'users' and 2 'non-users', were recruited from the Departments of Clinical Epidemiology \& Biostatistics and Medicine at McMaster University. Raters were stratified by user-status, defined as having familiarity with genetic association studies, i.e. if the rater routinely reads/ conducts genetic association studies. All four raters each rated the thirty studies for every item of the Q-Genie.

Item discrimination The extent to which each item distinguishes 'good' from 'bad' quality studies was assessed using item-total correlations. Items with item-total correlations below 0.2 or above 0.9 were considered uninformative and were candidates for exclusion from the tool [15].

Reliability Generalizability theory (G-theory) was used to establish inter-rater reliability (the extent to which a rating from one rater can be generalized to another), internal consistency (the extent to which a rating on one question can be generalized to another), inter-use reliability (the extent to which a rating from users can be generalized to non-users), and overall reliability. Formulas for the coefficients are presented in Additional file 1. All four raters, users and non-users, rated each study. Data from the ratings were used to ascertain G-coefficients, calculated separately for users and non-users, with the exception of inter-user reliability, for which data from both groups were used Raters used in this study were considered a random sample of all possible raters, and therefore we report absolute error G-coefficients.

Construct validity We tested the construct that high quality studies are cited more often and published in higher impact journals. These constructs were evaluated by testing their correlation with total score acquired on Q-Genie. We expected those studies acquiring higher scores on the Q-Genie tool to be published in journals with higher impact factors and cited more often than studies with lower scores on our tool. To account for the fact that some studies were published only in the preceding year and may not have had enough time to be cited, we assessed average citations per year as well as total citations. Additionally, we accounted for self-citation by excluding citations of the paper made by the first and senior authors, as this may artificially inflate the count and bias our assessment of validity. Citation count was ascertained using Web of Science (all databases). Correlation was determined using Spearman's $\rho$.

\section{Creating cut-points for low, moderate, and high quality on the Q-Genie tool}

In addition to the questions on Q-Genie, raters were given a question on global impression - "rate overall quality of the study". Ratings of 1 and 2 on this global impression question were classified as 'low', 3 and 4 as 'moderate', and 5-7 as 'high'. Borderline groups regression [17], a technique used to establish cut-points, was performed with total score on Q-Genie as the outcome and classification as 'low', 'moderate', or 'high' on the global impression question as the predictor. In this manner, total scores on Q-Genie corresponding to 'low', 'moderate', and 'high' on the global impression question were determined. The global impression question was only used to establish cut-points and is not part of QGenie.

\section{Empirical evaluation of the Q-Genie tool}

In addition to the psychometric assessment, we performed an empirical evaluation of the tool using published data from a meta-analysis investigating the association of CDKAL1 rs7754840 with type 2 diabetes [16]. Meta-analysis of this SNP contained significant heterogeneity and included seven datasets from six studies, making it conducive to this exercise. Characteristics of these studies are presented in Additional file 1: Table S2. We rated all six studies included in the meta-analysis of CDKAL1 rs7754840 using the Q-Genie tool. If the tool performed as anticipated, the effect estimate for this SNP should be more precise and less heterogeneous after exclusion of low quality studies, determined by QGenie, compared to the summary estimate ascertained using all studies. The $I^{2}$ statistic and Chi square test were used to establish heterogeneity.

Reliability analyses were conducted using G String IV (version 6.1.1). All other analyses were conducted on $R$ (version 3.0.2) and SPSS (version 20.0.0). 


\section{Results}

\section{Description of the final tool}

The final version of the Q-Genie tool contained 11 items (i.e. questions) marked on a 7-point Likert scale covering the following themes: scientific basis for development of the research question, ascertainment of comparison groups (i.e. cases and controls), technical and non-technical classification of genetic variant tested, classification of the outcome, discussion of sources of bias, appropriateness of sample size, description of planned statistical analyses, statistical methods used, test of assumptions in the genetic studies (e.g. agreement with the Hardy Weinberg equilibrium), and appropriate interpretation of results. The tool took approximately $20 \mathrm{~min}$ to complete per study.

\section{Psychometric assessment}

Item discrimination Item-total correlations (ITC) were calculated to determine the discrimination of each item (Tables 2 and 3 for users and non-users, respectively). As previously described, an ITC below 0.2 or above 0.9 are generally understood to be uninformative and the corresponding items are considered for exclusion [15]. Overall, no item had an ITC below 0.2 or above 0.9 for either group. The item with the lowest ITC (0.38) for users was question 2, which asked to "rate the study on the classification of the outcome (e.g. disease status or quantitative trait)". In contrast, question 1 had the lowest ITC for non-users (0.43).

A distribution of average ratings by group for each item is presented in Fig. 2. From the 11 items, item 1, which asked the rater to rate the study on the adequacy of the presented hypothesis and rationale, had the highest endorsement, understood as a rating of 6 or 7 on the 7-point scale, for both groups. On average, users endorsed this item $78 \%$ of the time and non-users endorsed it $60 \%$ of the time. Normally, high endorsement of a question may suggest that the question is not providing discriminative information about each study, since all studies tend to perform well on the item. We did not, however, exclude item 1 from our tool as it provides evidence of face validity and had an acceptable ITC in both groups.

Reliability Analysis of reliability was conducted using Gtheory. Inter-rater reliability, internal consistency, and overall reliability were assessed for users and non-users. Inter-rater reliability was 0.74 and 0.45 for users and nonusers, respectively. Internal consistency was similar in both groups (G-coefficient of 0.82 in users and 0.80 in non-users). Agreement between users and non-users was 0.64. Lastly, overall reliability, across raters and items, was 0.64 for users and 0.42 for non-users (Table 4).

Validity Spearman's $\rho$ for correlation between impact factor, average citations per year, and total citations, with total score on the Q-Genie tool are presented in Table 5. User scores had a stronger correlation with impact factor and average citations per year than non-user scores, although all values were above $\rho=0.30$. Total citations to date had the weakest correlation with scores on QGenie for both users and non-users (Spearman's $\rho=0.40$ and 0.33 for users and non-users, respectively), likely because total citations are confounded by time since publication. Spearman's $\rho$ did not change for either users or non-users when self-citations were excluded from the citation counts.

\section{Classification as low, moderate, or high quality from total score}

Borderline groups regression analysis indicated use of the following cut-points to designate low, moderate, and high quality studies for studies with case/control status as the outcome of interest: scores $\leq 35$ on the Q-Genie

Table 2 Item-total correlations and Cronbach's a if deleted for users

\begin{tabular}{|c|c|c|c|}
\hline Item & Question & $\begin{array}{l}\text { Item-total } \\
\text { correlation }\end{array}$ & $\begin{array}{l}\text { Cronbach's a if } \\
\text { item is deleted }\end{array}$ \\
\hline Question 1 & Please rate the study on the adequacy of the presented hypothesis and rationale. & 0.53 & 0.94 \\
\hline Question 2 & Please rate the study on the classification of the outcome (e.g. disease status or quantitative trait). & 0.38 & 0.94 \\
\hline Question 3 & Please rate the study on the description of comparison groups (e.g. cases and controls). & 0.51 & 0.94 \\
\hline Question 4 & Please rate the study on the technical classification of the exposure (i.e. the genetic variant). & 0.86 & 0.92 \\
\hline Question 5 & Please rate the study on the non-technical classification of the exposure (i.e. the genetic variant). & 0.55 & 0.94 \\
\hline Question 6 & Please rate the study on the disclosure and discussion of sources of bias. & 0.57 & 0.94 \\
\hline Question 7 & Please rate whether the study was adequately powered. & 0.84 & 0.93 \\
\hline Question 8 & Please rate the study on description of planned analyses. & 0.85 & 0.92 \\
\hline Question 9 & Please rate the study on the statistical methods. & 0.87 & 0.92 \\
\hline Question 10 & Please rate the study on the description and test of all assumptions and inferences. & 0.80 & 0.93 \\
\hline Question 11 & $\begin{array}{l}\text { Please rate the study on whether conclusions drawn by the authors were supported by the results } \\
\text { and appropriate methods. }\end{array}$ & 0.88 & 0.92 \\
\hline
\end{tabular}


Table 3 Item-total correlations and Cronbach's a if deleted for non-users

\begin{tabular}{|c|c|c|c|}
\hline Item & Question & $\begin{array}{l}\text { Item-total } \\
\text { correlation }\end{array}$ & $\begin{array}{l}\text { Cronbach's a if } \\
\text { item is deleted }\end{array}$ \\
\hline Question 1 & Please rate the study on the adequacy of the presented hypothesis and rationale. & 0.43 & 0.90 \\
\hline Question 2 & Please rate the study on the classification of the outcome (e.g. disease status or quantitative trait). & 0.53 & 0.89 \\
\hline Question 3 & Please rate the study on the description of comparison groups (e.g. cases and controls). & 0.51 & 0.89 \\
\hline Question 4 & Please rate the study on the technical classification of the exposure (i.e. the genetic variant). & 0.72 & 0.88 \\
\hline Question 5 & Please rate the study on the non-technical classification of the exposure (i.e. the genetic variant). & 0.56 & 0.89 \\
\hline Question 6 & Please rate the study on the disclosure and discussion of sources of bias. & 0.63 & 0.89 \\
\hline Question 7 & Please rate whether the study was adequately powered. & 0.76 & 0.88 \\
\hline Question 8 & Please rate the study on description of planned analyses. & 0.55 & 0.89 \\
\hline Question 9 & Please rate the study on the statistical methods. & 0.58 & 0.89 \\
\hline Question 10 & Please rate the study on the description and test of all assumptions and inferences. & 0.43 & 0.90 \\
\hline Question 11 & $\begin{array}{l}\text { Please rate the study on whether conclusions drawn by the authors were supported by the results } \\
\text { and appropriate methods. }\end{array}$ & 0.84 & 0.88 \\
\hline
\end{tabular}

tool indicate poor quality studies, $>35$ and $\leq 45$ indicate studies of moderate quality, and $>45$ indicate good quality studies (Fig. 3). Similarly, cut-points for studies without control groups (e.g. studies of quantitative traits) were created by excluding question 3 from the calculation of the total score on Q-Genie, since this question asked raters to assess the control group: scores $\leq 32$ on the Q-Genie tool indicate poor quality studies, $>32$ and $\leq 40$ indicate studies of moderate quality, and $>40$ indicate good quality studies. Applying these criteria to our sample of 30 studies revealed that 8 out of 30 studies were of poor quality (27\%), 17 were of moderate quality (56\%), and 5 were of high quality (17\%). Of the poor quality studies, a majority had biased technical and non-technical classification of the genetic variant $(50 \%$ had a score $<3$ and $100 \%$ had a score $<3$ on the respective question), inadequate disclosure of potential sources of bias (100\% had a score $<3$ ), inappropriate power ( $88 \%$ had a score $<3)$, poor statistical methods ( $75 \%$ had a score $<3)$, and inadequate testing of inferences $(63 \%$ had a score $<3)$.

\section{Empirical evaluation}

We applied the Q-Genie to an existing published metaanalysis of CDKAL1 rs7754840 [16]. After excluding studies with a score of $\leq 35$ on the Q-Genie tool, (those studies deemed to be of poor quality), the heterogeneity in meta-analysis was reduced from an $\mathrm{I}^{2}$ of $72 \%(95 \%$ CI $38 \%-87 \%$ ), Q-statistic of 21.1 (6 d.f.; p < 0.01) to an $\mathrm{I}^{2}$ of $0 \%$ (95\% CI $0 \%-75 \%$ ), Q-statistic of 3.04 (5 d.f.; $\mathrm{p}=0.69$ ) (Fig. 4). The summary effect size changed from 1.25 (95 \% CI 1.09-1.45) to 1.15 (95 \% CI 1.07-1.24). Although the difference between the two effect sizes is not statistically significant, the meta-analysis estimate after

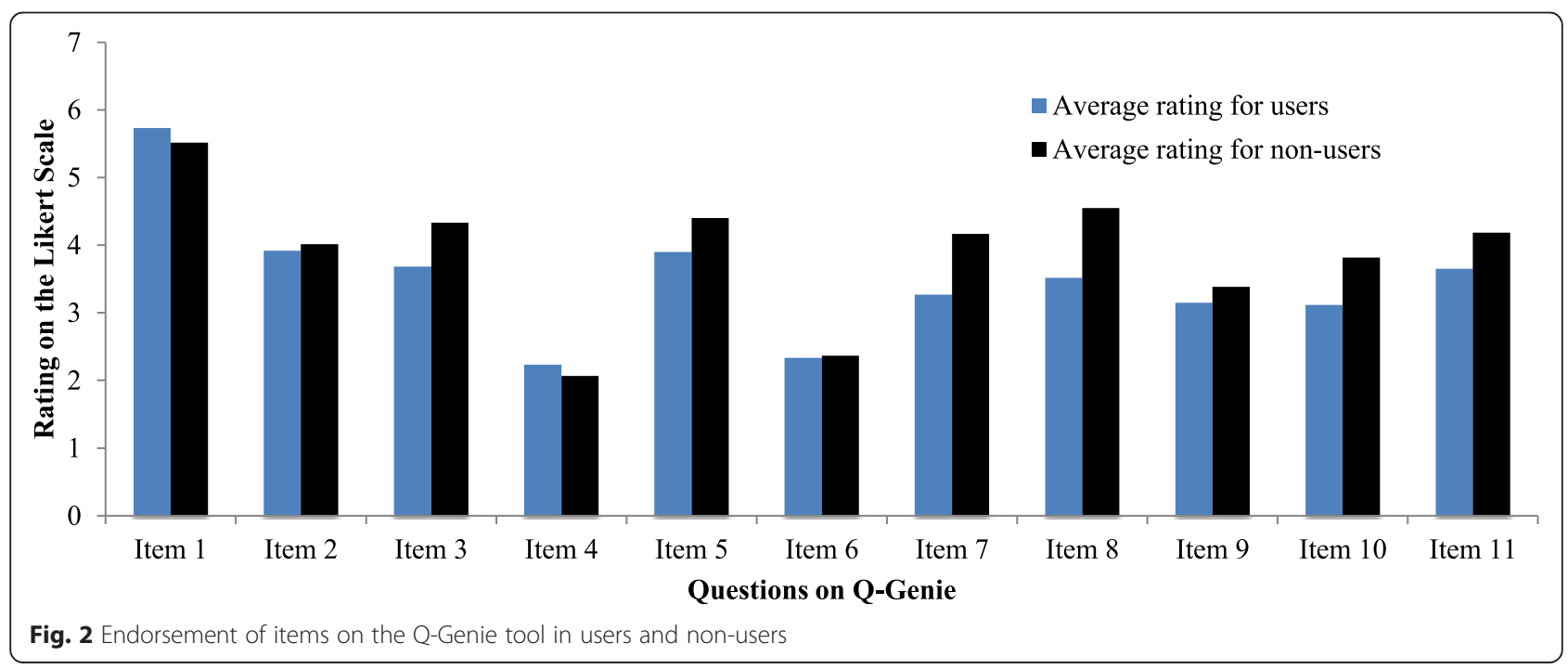


Table 4 G-coefficients of reliabilities, stratified by user-status

\begin{tabular}{lll}
\hline Reliability & $\begin{array}{l}\text { Users } \\
\left(\mathrm{n}_{\text {studies }}=30 ; \mathrm{n}_{\text {raters }}=2\right)\end{array}$ & $\begin{array}{l}\text { Non-users } \\
\left(\mathrm{n}_{\text {studies }}=30 ; \mathrm{n}_{\text {raters }}=2\right)\end{array}$ \\
\hline Internal consistency & 0.82 & 0.80 \\
Inter-rater & 0.74 & 0.45 \\
Overall & 0.64 & 0.42 \\
Inter-user* & 0.64 & \\
\hline
\end{tabular}

*all four raters were used to estimate this coefficient.

exclusion of the low quality study had tighter confidence intervals and is more precise.

\section{Discussion}

We have developed and validated a tool that assesses the global quality of published genetic association studies. The Q-Genie can be used to assess quality of genetic association studies in systematic reviews and meta-analyses, which can inform selection of studies for inclusion, examine the sensitivity of pooled effect sizes to indicators of study quality, and/or explain heterogeneity. The tool demonstrated good performance characteristics in a small sample of studies. Additionally, we applied our tool to a published systematic review of studies and found a decrease in heterogeneity and an increase in precision of estimates when used to exclude low quality studies.

Validity and Reliability We assessed the validity of the Q-Genie tool by measuring the correlation between predefined constructs, specifically impact factor, citations per year, and total citations with the total Q-Genie score, using Spearman's $\rho$. Our findings suggest that Q-Genie demonstrates construct validity in both groups, using measures of impact as a criterion. Other forms of validity should be tested in the future, including predictive, concurrent, as well as convergent and discriminative validity.

We used G-coefficients to estimate the reliability of the Q-Genie tool, which have previously been used to test other instruments in the psychometric literature

Table 5 Spearman's $\rho$ correlations of total scores on Q-Genie with impact factor and citation count, stratified by user-status

\begin{tabular}{|c|c|c|}
\hline Construct & $\begin{array}{l}\text { Users } \\
\left(\mathrm{n}_{\text {studies }}=30 ; \mathrm{n}_{\text {raters }}=2\right)\end{array}$ & $\begin{array}{l}\text { Non-users } \\
\left(n_{\text {studies }}=30 ; n_{\text {raters }}=2\right)\end{array}$ \\
\hline Impact factor & $0.61(p<0.01)$ & $0.45(p=0.02)$ \\
\hline $\begin{array}{l}\text { Average citations per } \\
\text { year }\end{array}$ & $0.51(p<0.01)$ & $0.38(p=0.04)$ \\
\hline $\begin{array}{l}\text { Average citations } \\
\text { (without self-cites) per } \\
\text { year }\end{array}$ & $0.52(p<0.01)$ & $0.39(p=0.03)$ \\
\hline Total citations to date & $0.40(p=0.03)$ & $0.33(p=0.08)$ \\
\hline
\end{tabular}

[18]. Our results show that Q-Genie is highly reliable in users (defined as those who read/conduct genetic association studies frequently) and moderately so in nonusers, which is not surprising since users presumably have a better understanding of quality in genetic association studies and are likely to agree more with each other than non-users. Additionally, data from studies in behavioral psychology suggest that people rate individual components based on intuitive impressions from global observations, and thus it appears logical that while different, both user and non-user estimates are reliable $[19,20]$. We expect that most users of Q-Genie will be experts in practice.

Empirical evaluation When the tool was applied to studies included in a meta-analysis of a well-known SNP associated with type 2 diabetes, we found that by excluding studies graded as poor quality by Q-Genie, we were able to substantially reduce heterogeneity and increase precision of the summary estimate. This furthers our confidence in the utility of the tool for use with systematic reviews and meta-analyses. Use of the tool may also encourage authors to explore other sources of heterogeneity, such as genetic heterogeneity, gene-environment interactions, and gene-gene interactions, if the possibility of between-study heterogeneity due to low-quality data is eliminated.

Limitations There are some limitations to our tool. Firstly, data from the four reviewers suggests that the tool takes approximately $20 \mathrm{~min}$ per study to complete, slightly longer for non-users (mean of $21 \mathrm{~min}, 15 \mathrm{~s}$ ) than users (mean of $18 \mathrm{~min}, 45 \mathrm{~s}$ ). Therefore, rating the quality of 30 studies included in a systematic review using Q-Genie would take about $10 \mathrm{~h}$. However, this estimate is comparable with time-to-complete measures of other tools in the literature, such as the Newcastle-Ottawa Scale and AMSTAR [21-23]. Additionally, once accustomed to the tool, raters likely become faster. Though the procedure may be time intensive, the gains in scientific rigor appear well worth the effort as demonstrated by application to the systematic review of the CDKAL1 SNP. Secondly, as with other global scoring tools, it is possible for a study to receive low scores on 2 dimensions, yet high scores on all others, and thus be considered a globally 'high quality' study. This can have limitations for answering specific research questions. However, because it is possible to obtain a score on each section using Q-Genie, users can be mindful of performance on each dimension. Lastly, because this is a pilot assessment with 30 studies and 4 reviewers, additional testing is warranted to gain support for our findings.

Q-Genie is available for download from <http:// fhs.mcmaster.ca/pgp/links.html>. We note that our tool 


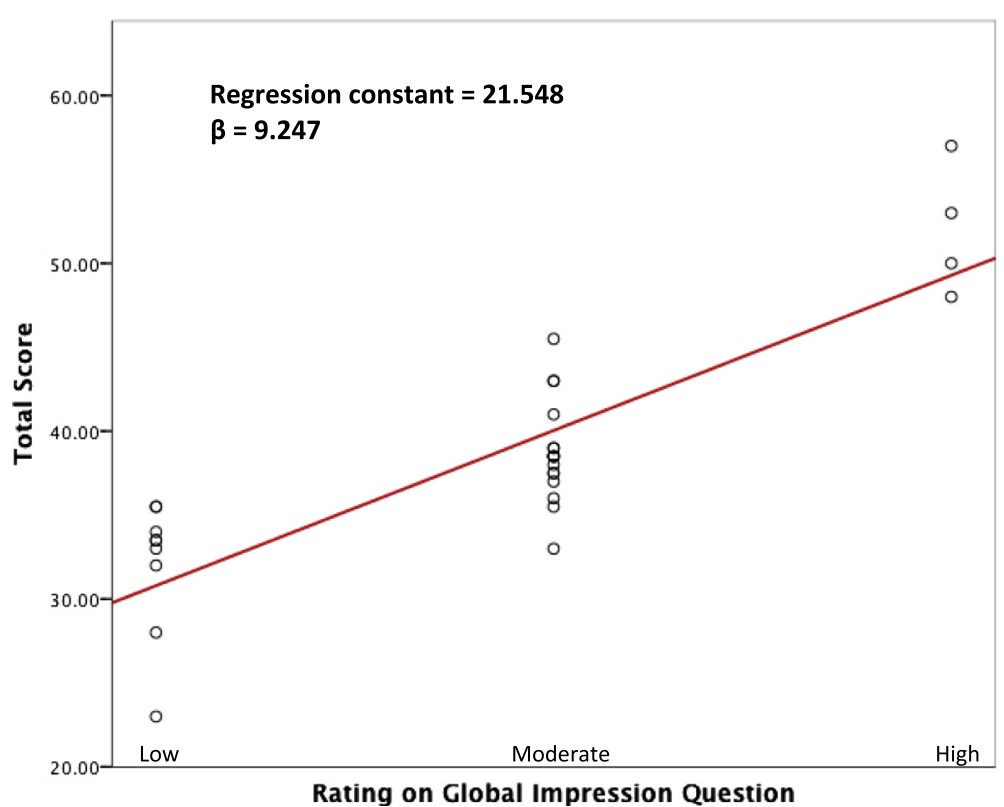

Fig. 3 Plot of borderline groups regression depicting total scores on the Q-Genie tool corresponding with 'low' , 'moderate', and 'high' quality of genetic association study

would benefit from testing in a larger sample of studies as well as an assessment of additional measures of validity, and we encourage other groups to further test our tool. We also welcome comments that can be used to inform revisions of the tool.

\section{Conclusions}

Based on our evaluation of 30 studies from a published systematic review, it appears that many publications in literature may be of poor quality despite published guidelines designed to improve the quality of genetic

CDKAL1

rs7754840 (C)

Study

Rees Diabetologia 2011

Chauhan Diabetes 2010

Chauhan Diabetes 2010

Chidambaram Met 2010

Sanghera BMC 2008

Anand Diabetes Care 2013

Sim PLoS Genet 2011

Summary

Het:

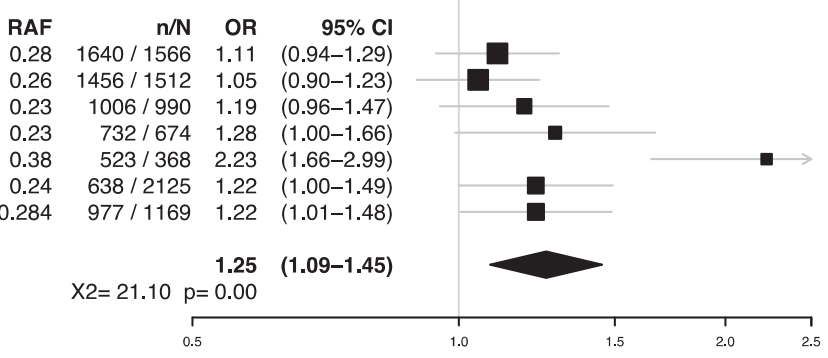

CDKAL1

rs7754840 (C)

Study

Rees Diabetologia 2011

Chauhan Diabetes 2010

Chauhan Diabetes 2010

Chidambaram Met 2010

Anand Diabetes Care 2013

Sim PLoS Genet 2011

Summary

Het:

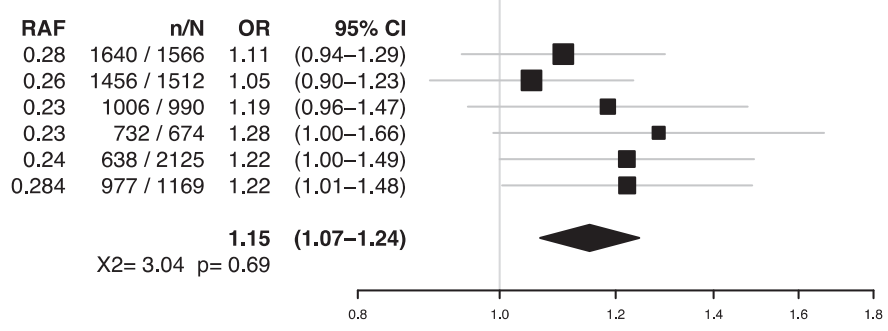

$\mathrm{RAF}=$ Risk allele frequency; $\mathrm{OR}=$ odds ratio; $\mathrm{n} / \mathrm{N}$ indicate sample size for cases and controls, respectively

Fig. 4 Forest plot of CDKAL1 rs7754840 with and without exclusion of low quality studies 
association studies. The Q-Genie tool was developed and validated to facilitate the assessment of published studies and to ultimately identify high quality studies when planning meta-analyses of genetic association studies. Integration of our tool into systematic reviews and meta-analyses can help improve the state of evidence in the field of genetic epidemiology, which is currently plagued with irreproducible findings. Our data shows that Q-Genie demonstrates good inter-rater reliability, internal consistency, and overall reliability. We encourage using the Q-Genie tool as it can substantially increase the quality of meta-analyses in genetic association studies.

\section{Additional file}

Additional file 1: Supplementary Box 1 Formulas for absolute error $\mathrm{G}$-coefficients for reliability. Figure S1. Frequency plot of increase in publication of genetic association studies (determined via a search of PubMed). Table S1. Description of studies used for psychometric assessment of Q-Genie. Table S2. Description of studies included in the meta-analysis of CDKAL1 rs7754840 [16] used in the empirical evaluation of Q-Genie.

\section{Competing interests}

The authors report no competing interests.

\section{Authors' contributions}

ZNS designed the study, was involved in data collection, analysis and interpretation of data, and was involved in drafting and critically revising the manuscript. DM was involved in designing the study and revising the manuscript. RJD was involved in analysis and interpretation of data, as well as revising the manuscript. PGJ was involved in study design, data collection, as well as revising the manuscript. MG was involved in study design, data collection, as well as revising the manuscript. BBD was involved in data collection and revising the manuscript. GN was involved in study design, analysis and interpretation of data, as well as revising the manuscript. SSA was involved in study design, interpretation of data, as well as drafting and revising the manuscript. All authors read and approved the final manuscript.

\section{Acknowledgement}

We would like to acknowledge Drs. Guillaume Pare, Meghan McConnell, and Sebastien Robiou-du-Pont for the guidance in developing the tool.

\section{Funding}

ZNS is supported by the Ontario Graduate Scholarship and the Canadian Diabetes Association Doctoral Award. SSA holds the Heart and Stroke Foundation of Ontario Michael G. DeGroote endowed Chair in Population Health and a Canada Research Chair in Ethnicity and Cardiovascular Disease. DM holds a Canada Research Chair in Genetics of Obesity. GN holds a Canada Research Chair in Cognitive Dimensions of Learning.

\section{Author details}

${ }^{1}$ Population Genomics Program, Department of Clinical Epidemiology and Biostatistics, McMaster University, Hamilton, ON, Canada. ${ }^{2}$ Chanchlani Research Centre, McMaster University, Hamilton, ON, Canada. ${ }^{3}$ Department of Pathology and Molecular Medicine, McMaster University, Hamilton, ON, Canada. ${ }^{4}$ Department of Medicine, McMaster University, 1280 Main St West, Hamilton, ON L8S 4L8, Canada. ${ }^{5}$ Department of Medicine, Western University, London, ON, Canada. ${ }^{6}$ Programme for Educational Research and Development (PERD), McMaster University, Hamilton, ON, Canada.

\section{References}

1. Hattersley AT, McCarthy MI. What makes a good genetic association study? Lancet. 2005;366:1315-23.

2. Ioannidis JPA, Boffetta P, Little J, O'Brien TR, Uitterlinden AG, Vineis P, et al. Assessment of cumulative evidence on genetic associations: interim guidelines. Int J Epidemiol. 2008;37:120-32.

3. Little J, Higgins JPT, loannidis JPA, Moher D, Gagnon F, von Elm E, et al. STrengthening the REporting of Genetic Association Studies (STREGA): an extension of the STROBE statement. PLoS Med. 2009;6:e22.

4. Li A, Meyre D. Challenges in reproducibility of genetic association studies: lessons learned from the obesity field. Int J Obes (Lond). 2013;37:559-67.

5. Janssens ACJW, loannidis JPA, van Duijn CM, Little J, Khoury MJ. Strengthening the reporting of genetic risk prediction studies: the GRIPS statement. BMJ. 2011;342:d631.

6. Freimer N, Sabatti C. The use of pedigree, sib-pair and association studies of common diseases for genetic mapping and epidemiology. Nat Genet. 2004:36:1045-51.

7. Freimer NB, Sabatti C. Guidelines for association studies in Human Molecular Genetics. Hum Mol Genet. 2005;14:2481-3.

8. Guidelines for Genetic Association Studies in Diabetologia [http:// www.diabetologia-journal.org/genetics\%20guidelines.htm]

9. Regehr G, Freeman R, Robb A, Missiha N, Heisey R. OSCE performance evaluations made by standardized patients: comparing checklist and global rating scores. Acad Med. 1999;74(10 Suppl):S135-7.

10. Bick JS, Demaria S, Kennedy JD, Schwartz AD, Weiner MM, Levine Al, et al. Comparison of expert and novice performance of a simulated transesophageal echocardiography examination. Simul Healthc. 2013;8:329-34.

11. Hodges B, Regehr G, McNaughton N, Tiberius R, Hanson M. OSCE checklists do not capture increasing levels of expertise. Acad Med. 1999;74:1129-34.

12. Ramos KD. Validation of the Fresno test of competence in evidence based medicine. BMJ. 2003;326:319-21.

13. Yule S, Rowley D, Flin R, Maran N, Youngson G, Duncan J, et al. Experience matters: comparing novice and expert ratings of non-technical skills using the NOTSS system. ANZ J Surg. 2009;79:154-60.

14. Nature Genetics Editorial Board. Framework for a fully powered risk engine. Nat Genet 2005, 37:1153.

15. Streiner DL, Norman GR. Health Measurement Scales: A Practical Guide to Their Development and Use [Paperback]. Fourthth ed. Toronto: Oxford University Press; Fourth Edition edition; 2008.

16. Sohani ZN, Deng WQ, Pare G, Meyre D, Gerstein HC, Anand SS. Does genetic heterogeneity account for the divergent risk of type 2 diabetes in South Asian and white European populations? Diabetologia 2014;57(11):2270-81. doi:10.1007/s00125-014-3354-1.

17. Wood TJ, Humphrey-Murto SM, Norman GR. Standard setting in a small scale OSCE: a comparison of the Modified Borderline-Group Method and the Borderline Regression Method. Adv Health Sci Educ Theory Pract. 2006;11:115-22.

18. Eva KW, Reiter Hl, Rosenfeld J, Norman GR. The relationship between interviewers' characteristics and ratings assigned during a multiple mini-interview. Acad Med. 2004;79:602-9.

19. Ambady N. The Perils of Pondering: Intuition and Thin Slice Judgments. Psychol Inq. 2010;21:271-8.

20. Wood TJ. Exploring the role of first impressions in rater-based assessments. Adv Health Sci Educ Theory Pract 2014;19(3):409-27. doi:10.1007/s10459-013-9453-9.

21. Shea BJ, Hamel C, Wells GA, Bouter LM, Kristjansson E, Grimshaw J, et al. AMSTAR is a reliable and valid measurement tool to assess the methodological quality of systematic reviews. J Clin Epidemiol. 2009;62:1013-20.

22. Stang A. Critical evaluation of the Newcastle-Ottawa scale for the assessment of the quality of nonrandomized studies in meta-analyses. Eur J Epidemiol. 2010:25:603-5.

23. The Newcastle-Ottawa Scale (NOS) for assessing the quality of nonrandomised studies in meta-analyses [http://www.ohri.ca/programs/clinical_epidemiology/ oxford.asp] 